\title{
Paramedian approach: a better technique for spinal anaesthesia in elderly patients for proximal femoral fracture surgery
}

\author{
T.Zhurda, R. Sturce, M.Bajraktari, S. Horeshka, E.Jaho, B. Dautaj \\ University Hospital of Trauma, Department of Anesthesiology, Tirana, Albania
}

Background and Goal of Study

Spinal anaesthesia (SA) has been the technique of choice for femur fracture surgery in elderly patients. Usually SA is performed using the midline approach. But this approach is technically difficult where adequate flexion for proper positioning is not possible, especially in elderly patients with proximal femur fracture because they have sever the pain and degenerative changes in the structural components of their spine. For these reasons, some authors have recommended paramedian approach because this approach does not require flexed position as in midline approach. The goal of this study was to determine which of approaches of SA should be preferred as first choice in elderly patients for proximal femur fracture surgery.

\section{Materials and Methods}

We prospectively studied 88 patients of either sex, aged >65 years, who underwent open repair of proximal femur fracture surgery.These patients were randomized to receive SA either with the midline approach (group M, n=44) or paramedian approach (group PM, n=44).

Under all aseptic conditions, the SA was given with 25 gauge spinal needle in sitting position at L3-L4 interspace. In both groups, $0.5 \%$ isobaric bupivacaine 2.5 to $3 \mathrm{ml}$ was injected according to the type of surgery. In paramedian approach, skin wheal was raised $1.5 \mathrm{~cm}$ lateral to the inferior aspect of superior spinous process and needle was directed and advanced at 10-25 degree angle towards the midline.For every patient we considered the: average age, success rate in first attempt, number of punctions and failure.

\section{Results}

Age of the patients varied between 65 and 92 years. The M group had 24 females and 21 males. The PM group had 21 females and 23 males. The mean age in the M group was 71.4 and in the PM group 73.2 years. (Tab.1)

First attempt success rate was $61.3 \%$ (27 patients) of the M group and $90.9 \%$ (40 patients) of the PM group. More than one attempt was required in 21 patients (47.7\%) of the $\mathrm{M}$ group, and 6 patients (13.6\%) of the PM group. In three cases $(6.81 \%)$ of the M group, spinal puncture was impossible and were successful with the paramedian approach. In both the groups ,there was no failure of SA. (Tab.2)

\begin{tabular}{|l|l|l|}
\hline \multicolumn{3}{|c|}{ Tab.1 Demographic characteristics } \\
\begin{tabular}{|l|l|l|}
\hline Characteristics & $\begin{array}{c}\text { Group M } \\
\text { n=44 }\end{array}$ & $\begin{array}{l}\text { Group PM } \\
\text { n=44 }\end{array}$ \\
\hline Age (years) & 71.4 & 73.2 \\
\hline Sex (F\M) & $24 / 21$ & $21 / 23$ \\
\hline
\end{tabular}
\end{tabular}

Tab.2 Numbers of attempts for spinal anaesthesia

\begin{tabular}{|l|l|l|}
\hline Number of attempts & $\begin{array}{l}\text { Group M } \\
\mathbf{n = 4 4}\end{array}$ & $\begin{array}{l}\text { Group PM } \\
\mathbf{n = 4 4}\end{array}$ \\
\hline First attempt & $27(61.3 \%)$ & $\mathbf{4 0}(\mathbf{9 0 . 9 \% )}$ \\
\hline More then one attempt & $21(47.7 \%)$ & $\mathbf{6}(13.6 \%)$ \\
\hline Impossible spinal puncture & $3(6.81 \%)$ & 0 \\
\hline Failure & 0 & 0 \\
\hline
\end{tabular}

\section{Discussion}

Proper positioning is the key to making the SA quick and successful. In certain conditions like obesity, spinal deformity or fracture it is very difficult to make proper position and administration of SA through median approach is difficult. SA by the classic median approach in which the needle traverses the entire supraspinous and interspinous ligaments, is usually unsuccessful in elderly patients with proximal femur fracture because they have sever the pain and degenerative changes in their spine, which can make adequate flexion of the back not possible and interspinous spaces may not open up. An alternative approach is paramedian approach which does not require flexed posture as in median approach and needle placement through para spinal muscle mass is easy and the anatomical limitation of the spinuos process is avoided. In our study, paramedian approach was better as regards to success rate with the first attempt compared to median approach. Influence of technique of SA on success rate is determined by a number of studies and almost all of the studies favor paramedian approach.

\section{Conclusion}

In conclusion, paramedian approach is better approach for SA and should be routinely used as first choice in elderly patients with proximal femur fracture where adequate flexion for proper positioning for midline approach is not possible.

\section{Reference}

1.Ahsan-ul-haq M, Amin S, Javaid S. Paramedian technique of spinal anesthesia in elderly patients for hip fracture surgery. J Coli Physicians Surg Pak. 2005; 15: 160-1. 2.Ahmed T, Ullah H. Paramedian technique of spinal anaesthesia in elderly patients for hip fracture surgery. J Coll Physicians SurgPak. 2007;17(3):184. 